\title{
JAK2 promotes brown adipose tissue function and is required for diet- and cold-induced thermogenesis in mice
}

\author{
Sally Yu Shi ${ }^{1,2}$ - Wei Zhang ${ }^{1,3}$ - Cynthia T. Luk ${ }^{1,2}$ - Tharini Sivasubramaniyam ${ }^{1,2}$ • \\ Jara J. Brunt ${ }^{1,2} \cdot$ Stephanie A. Schroer ${ }^{1}$ - Harsh R. Desai ${ }^{1,4}$ - Alexandra Majerski ${ }^{1}$. \\ Minna Woo ${ }^{1,2,5}$
}

Received: 19 August 2015 / Accepted: 28 September 2015 /Published online: 29 October 2015

(C) Springer-Verlag Berlin Heidelberg 2015

\begin{abstract}
Aims/hypothesis Non-shivering thermogenesis in adipose tissue can be activated by excessive energy intake or following cold exposure. The molecular mechanisms regulating this activation have not been fully elucidated. The Janus kinase (JAK) - signal transducer and activator of transcription (STAT) pathway mediates the signal transduction of numerous hormones and growth factors that regulate adipose tissue development and function, and may play a role in adaptive thermogenesis. Methods We analysed mRNA and protein levels of uncoupling protein 1 (UCP1) and JAK2 in different adipose depots in response to metabolic and thermal stress. The in vivo role of JAK2 in adaptive thermogenesis was examined using mice with adipocyte-specific Jak2 deficiency (A-Jak2 KO). Results We show in murine brown adipose tissue (BAT) that JAK2 is upregulated together with UCP1 in response to high-
\end{abstract}

Electronic supplementary material The online version of this article (doi:10.1007/s00125-015-3786-2) contains peer-reviewed but unedited supplementary material, which is available to authorised users.

Minna Woo

mwoo@uhnresearch.ca

1 Toronto General Research Institute, 101 College Street, MaRS Centre/TMDT, Toronto, ON, Canada M5G 1L7

2 Institute of Medical Science, University of Toronto, Toronto, ON, Canada

3 Present address: Department of Endocrinology, Shandong Provincial Qianfoshan Hospital, Shandong University, Jinan, China

4 Department of Laboratory Medicine and Pathobiology, University of Toronto, Toronto, ON, Canada

5 Division of Endocrinology, Department of Medicine, Toronto General Hospital, University Health Network, University of Toronto, Toronto, ON, Canada fat diet (HFD) feeding and cold exposure. In contrast to white adipose tissue, where JAK2 was dispensable for UCP1 induction, we identified an essential role for BAT JAK2 in diet- and cold-induced thermogenesis via mediating the thermogenic response to $\beta$-adrenergic stimulation. Accordingly, A-Jak2 $\mathrm{KO}$ mice were unable to upregulate BAT UCP1 following a HFD or after cold exposure. Therefore, A-Jak $2 \mathrm{KO}$ mice were cold intolerant and susceptible to HFD-induced obesity and diabetes.

Conclusions/interpretation Taken together, our results suggest that JAK2 plays a critical role in BAT function and adaptive thermogenesis. Targeting the JAK-STAT pathway may be a novel therapeutic approach for the treatment of obesity and related metabolic disorders.

Keywords Adaptive thermogenesis - Brown adipose tissue · Diabetes · Glucose homeostasis · Janus kinase 2 (JAK2) · Obesity $\cdot$ Uncoupling protein 1 (UCP1)

$\begin{array}{ll}\text { Abbreviations } \\ \text { BAT } & \begin{array}{l}\text { Brown adipose tissue } \\ \text { CLAMS }\end{array} \\ \text { Comprehensive laboratory animal monitoring } \\ \text { system }\end{array}$


STAT Signal transducer and activator of transcription

VAT Visceral adipose tissue

UCP1 Uncoupling protein 1

$\dot{V} \mathrm{O}_{2} \quad$ Oxygen consumption volume

\section{Introduction}

The expanding global epidemic of obesity necessitates a better understanding of its underlying pathogenic mechanisms and the development of novel treatment strategies. Obesity is driven by an imbalance between caloric intake and energy expenditure, with excess nutrients stored as body fat [1]. While adipose tissue is best known for its function in fuel storage, specialised adipocytes can promote energy expenditure and suppress weight gain via non-shivering thermogenesis [2, 3]. Brown adipocytes, which are located in dedicated depots, express constitutively high levels of uncoupling protein 1 (UCP1) that uncouples the electron transport chain, leading to the generation of heat at the expense of ATP production [4]. In contrast, beige adipocytes, which also express UCP1, develop in white adipose tissue in response to thermogenic activators [5]. Recent studies in humans indicate that brown/beige adipocytes are crucial in the regulation of energy expenditure. Importantly, their activity is inversely correlated with severity of the metabolic syndrome [6-8]. Thus, strategies to enhance brown or beige adipocyte function represent an appealing approach to combating obesity.

Thermogenesis in brown adipose tissue (BAT) is activated by excessive caloric intake or cold exposure to counteract the energy surplus or maintain body temperature, respectively [9, 10]. Cold- and diet-induced thermogenesis are mediated by the sympathetic nervous system through stimulation of $\beta_{3}$-adrenergic receptors on BAT to induce UCP1 activity and/or content $[11,12]$. Cold also activates beige adipocyte development [5]. A better understanding of the molecular mechanisms regulating adaptive thermogenesis would facilitate the development of brown or beige adipocyte activators for the treatment of metabolic diseases.

The Janus kinase (JAK) - signal transducer and activator of transcription (STAT) pathway is activated by a variety of cytokines, hormones and growth factors and plays an important role in many cellular functions [13]. Over the past few years, emerging evidence has linked JAK-STAT signalling to brown and beige adipocyte biology [14-16]. Nevertheless, the specific role of JAK2 (a ubiquitously expressed JAK family member) in these processes has not been addressed.

JAK2 is activated in response to a number of cytokines and hormones that regulate brown and beige adipocytes such as growth hormone (GH) [17, 18], prolactin [19], leptin [20, 21], IL-6 [22, 23] and ciliary neurotrophic factor (CNTF) [24]. In this study, we investigated the role of JAK2 in thermogenesis, particularly in response to metabolic and thermal stress. We show that JAK2 mediates the thermogenic response to $\beta_{3}$-adrenergic stimulation and is required for diet- and coldinduced UCP1 in BAT. Accordingly, mice with an adipose tissue specific Jak2 deficiency (herein referred to as A-Jak2 $\mathrm{KO}$ mice) were unable to maintain body temperature in response to cold exposure and had exacerbated obesity, insulin resistance and glucose intolerance after a high fat-diet (HFD).

\section{Methods}

Animal model A-Jak2 $\mathrm{KO}$ mice in which the Cre gene is driven by the adipocyte protein 2 (Ap2) promoter (Ap2Cre $\left.J a k 2^{f l / f l}\right)$ were described previously [25]. Jak2 ${ }^{\mathrm{fl} / \mathrm{fl}}$ mice $\left(J a k 2^{t m 1 K u w}\right)$ were kindly provided by K.-U. Wagner (University of Nebraska Medical Center, Omaha, NE, USA) $[26,27]$. Ap2 $\mathrm{Cre}^{+} \mathrm{Jak2}^{+/+}$littermates served as controls, and mice were maintained on a mixed $129 \mathrm{~Sv}$ and C57BL/6 background. Animals were housed in a temperature controlled and pathogen free facility at the Toronto Medical Discovery Tower (Toronto, ON, Canada) with a 12 h light-dark cycle and free access to water and standard irradiated rodent chow (5\% fat; Harlan Teklad, Indianapolis, IN, USA). At 8 weeks of age, a cohort of mice was randomly selected to receive an HFD (60\% fat, $24 \%$ carbohydrates and $16 \%$ protein based on calorie content; F3282; Bio-Serv, Flemington, NJ, USA) for 8 10 weeks. Ambient temperature was maintained at $20^{\circ} \mathrm{C}$ unless indicated otherwise. All animal experimental protocols were approved by the Toronto General Research Institute Animal Care Committee.

In vivo metabolic analyses In vivo metabolic analyses were performed as previously described [28-30]. For insulin signalling experiments, female mice fasted overnight were i.p. injected with insulin lispro (B28Lys,B29Pro human insulin; $5 \mathrm{U} / \mathrm{kg}$; Humalog, Eli Lilly, Indianapolis, IN, USA) or PBS. Tissues were removed $10 \mathrm{~min}$ later and snap frozen. To measure energy expenditure, mice were individually housed in a comprehensive laboratory animal monitoring system (CLAMS; Columbus Instruments, Columbus, OH, USA) with free access to food and water. After $24 \mathrm{~h}$ acclimatisation, data were collected for $24 \mathrm{~h}$. Physical activity was determined by infrared beam breaks in the $x$ and $z$ axes during a single measurement interval. Investigators were not blinded to group assignment.

$\boldsymbol{\beta}_{\mathbf{3}}$-adrenergic agonist treatment The effect of a $\beta_{3}$-adrenergic agonist on the metabolic rate was assessed as previously described [31, 32]. Briefly, chow-fed mice were placed in a CLAMS system and allowed to acclimatise. Baseline data were collected for $2 \mathrm{~h}$ on the morning of the experiment. Thereafter, CL316,243 (Sigma, St. Louis, MO, USA; dissolved in $0.9 \% \mathrm{NaCl}$ ), a selective $\beta_{3}$-adrenergic agonist, was 
injected intraperitoneally at a dose of $1.0 \mathrm{mg} / \mathrm{kg}$ body weight. Metabolic data were collected for another $3 \mathrm{~h}$.

Body temperature and cold exposure Body temperature was measured in fed mice between 10:00 and 11:00 hours using a rectal temperature probe. For cold exposure experiments, mice were housed individually at an ambient temperature of $4^{\circ} \mathrm{C}$ for $6 \mathrm{~h}$.

Analyses of serum variables Serum cytokine levels were determined in overnight-fasted animals using the Milliplex Mouse Serum Adipokine Kit (Millipore, Billerica, MA, USA).

Histology Tissues were harvested, fixed in 4\% paraformaldehyde in $0.1 \mathrm{~mol} / 1 \mathrm{PBS}$ (pH 7.4) and processed to form paraffin blocks. Adipocyte size was measured in haematoxylin and eosin (H\&E) stained slides using cellSens software (Olympus, Tokyo, Japan). Immunohistochemical analysis was performed on BAT sections using a rabbit anti-mouse UCP1 antibody (1:500 dilution; Abcam, Cambridge, UK).

Citrate synthase activity Citrate synthase (CS) activity in adipose tissue homogenates was measured using a CS assay kit (Sigma) according to the manufacturer's instructions and normalised to protein content.

RNA isolation and quantitative RT-PCR RNA was isolated using Trizol (Invitrogen, Carlsbad, CA, USA) and reverse transcribed to cDNA with random primers using the M-MLV enzyme (Invitrogen). Quantitative real-time PCR was performed using specific primers and SYBR Green master mix on a 7900HT Fast-Real-Time PCR System (Applied Biosystems, Carlsbad, CA, USA). Each sample was run in triplicate. The relative mRNA abundance of each gene was normalised to the expression level of the housekeeping gene Gapdh or 18S.

Immunoblotting Tissues were mechanically homogenised in ice-cold lysis buffer and centrifuged at $14,000 \mathrm{~g}$ for $10 \mathrm{~min}$ at $4^{\circ} \mathrm{C}$. The resulting supernatant fraction was separated by SDSPAGE, and immunoblotted with antibodies against UCP-1 (1:500), actin $(1: 1,000)$ (Santa Cruz Biotechnology, Santa Cruz, CA, USA), phospho-Akt (S473) (1:500), total Akt $(1: 1,000)$, phospho-STAT3 (Y705) $(1: 500)$, total STAT3 $(1: 1,000)$, phospho-STAT5 (Y694) $(1: 500)$, total STAT5 $(1: 1,000)$, total JAK2 $(1: 1,000)$, and glyceraldehyde-3phosphate dehydrogenase (GAPDH; 1:5,000) (Cell Signaling Technology, Danvers, MA, USA).

Statistical analysis The criterion for exclusion of any data point was a distance greater than $2.5 \times \mathrm{SD}$ from the mean of the group. Data are presented as the mean \pm SEM. Values were analysed by two-tailed independent sample Student's $t$ test or one-way ANOVA followed by Tukey's post hoc test, as appropriate, using GraphPad Prism version 5 (GraphPad Software, La Jolla, CA, USA). Comparison of energy expenditure as a function of body mass between genotype groups was analysed by ANCOVA. A value of $p<0.05$ was considered statistically significant.

\section{Results}

Adipocyte JAK2 expression and diet-induced thermogenesis To better understand the molecular mechanisms regulating energy expenditure in adipose tissue, we first investigated the thermogenic programme of different fat depots in response to caloric excess. Feeding mice a HFD for $8-10$ weeks elevated the daily energy intake by approximately $20 \%$ (Fig. 1a) and significantly increased body weight (Fig. 1b). As expected, this disruption in energy balance significantly upregulated UCP1 mRNA and protein levels in BAT (Fig. 1c, d). Furthermore, HFD induced UCP1 in white adipose tissue, particularly the subcutaneous adipose tissue (SAT) depot (Fig. 1c, d), which is abundant in beige adipocyte precursors [5]. Of note, levels of $U c p 1$ in SAT and visceral adipose tissue (VAT) were two to three orders of magnitude lower than in BAT. Consistent with UCP1 upregulation, the HFD also significantly elevated the expression of other brown/beige adipocyte markers in BAT and SAT (electronic supplementary material [ESM] Fig. 1a-c).

We next examined JAK2 expression in different fat depots. Interestingly, Jak2 was more abundantly expressed in BAT than in white adipose tissue (Fig. 1e), suggesting that it has a role in BAT biology. Intriguingly, along with UCP1 induction, we observed an increase in JAK2 mRNA and protein abundance in response to the HFD (Fig. 1f, g). Similar to UCP1, the extent of JAK2 induction was greater in BAT and SAT than VAT. Together, these results suggest that JAK2 may play a role in diet-induced thermogenesis.

Impaired BAT function in A-Jak2 KO mice To test whether adipose JAK2 has an essential regulatory role in adaptive thermogenesis, we used mice with an adipocyte-specific $J a k 2$ deficiency (A-Jak2 KO) that we had generated previously [25]. Given the pivotal role of $\beta$-adrenergic signalling in both diet- and cold-induced thermogenesis [11, 12], we first tested the impact of Jak2 deficiency on the thermogenic response to adrenergic stimulation by monitoring the metabolic rate before and after injection of the selective $\beta_{3}$-adrenergic agonist CL316,243. CL316,243 treatment induced a rapid elevation in energy expenditure in control mice (Fig. 2a). However, this response was significantly blunted in A-Jak2 $\mathrm{KO}$ mice, suggesting that adipose JAK2 is required for the full 

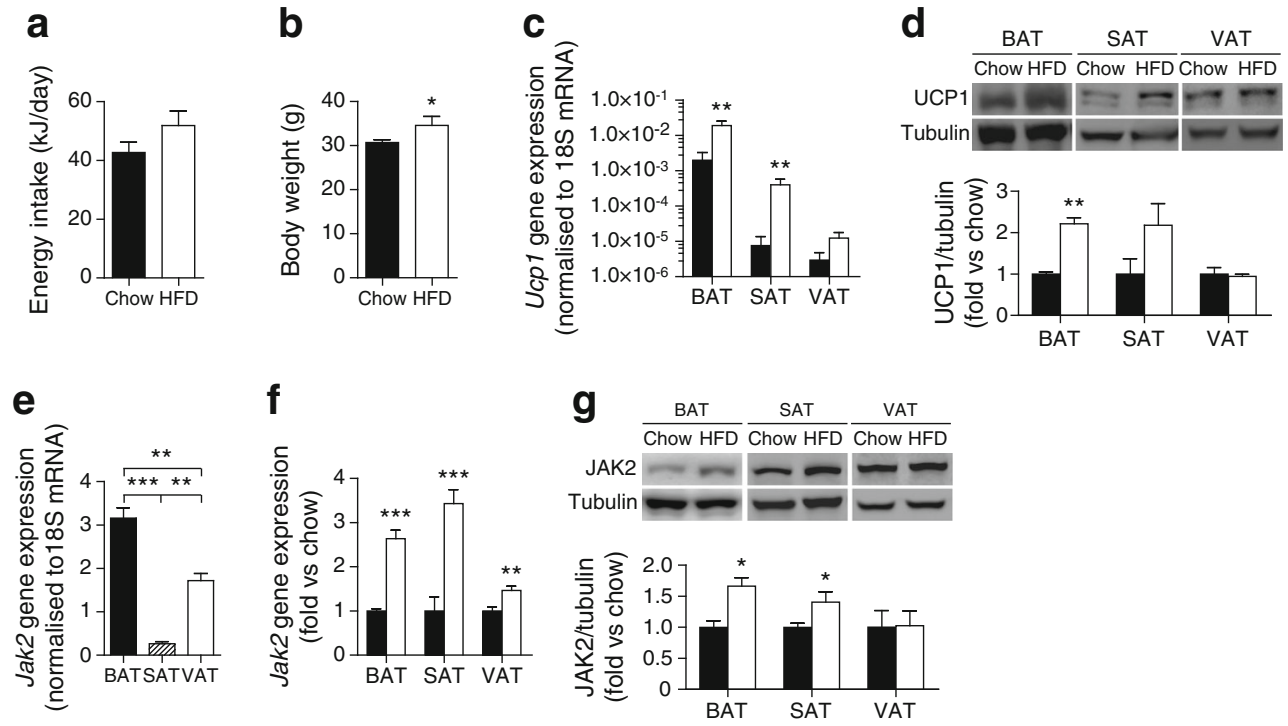

Fig. 1 Energy excess concomitantly upregulates UCP1 and JAK2 expression in mouse adipose tissue. (a) Daily energy intake and (b) body weight in chow- and HFD-fed mice $(n \geq 9)$. (c, d) Expression of UCP1 (c) mRNA $(n \geq 5)$ and (d) protein $(n=3)$ in adipose tissues of chow- and HFD-fed mice. Note that the $y$-axis in (c) is plotted on a $\log _{10}$ scale.

activation of thermogenesis in response to adrenergic stimulation.

Indeed, consistent with impaired BAT function, our previous work showed extensive adipose expansion and the development of progressive obesity in A-Jak2 $\mathrm{KO}$ mice even on a chow diet [25]. At 1 month of age, when there was no difference in body weight, A-Jak2 $\mathrm{KO}$ mice had decreased BAT Ucp1 mRNA abundance that was associated with lower
Black, chow fed; white; HFD fed. (e) Expression of Jak2 mRNA in chow-fed mice $(n \geq 3)$. (f, g) Expression of JAK2 (f) mRNA $(n \geq 5)$ and $(\mathbf{g})$ protein $(n=3)$ in adipose tissues of chow- and HFD-fed mice. Results are means \pm SEM. ${ }^{*} p<0.05,{ }^{* *} p<0.01$ and ${ }^{* * *} p<0.001$

whole-body energy expenditure (Fig. 2b, c). Similar trends were also observed in older animals (Fig. 2d, e). H\&E staining of BAT sections indicated the presence of larger adipocytes with bigger fat droplets in 1-month-old AJak2 KO mice (Fig. 2e). In older mice, this abnormality in adipocyte morphology was more pronounced with the appearance of large unilocular fat droplets inside the cells (Fig. 2e).
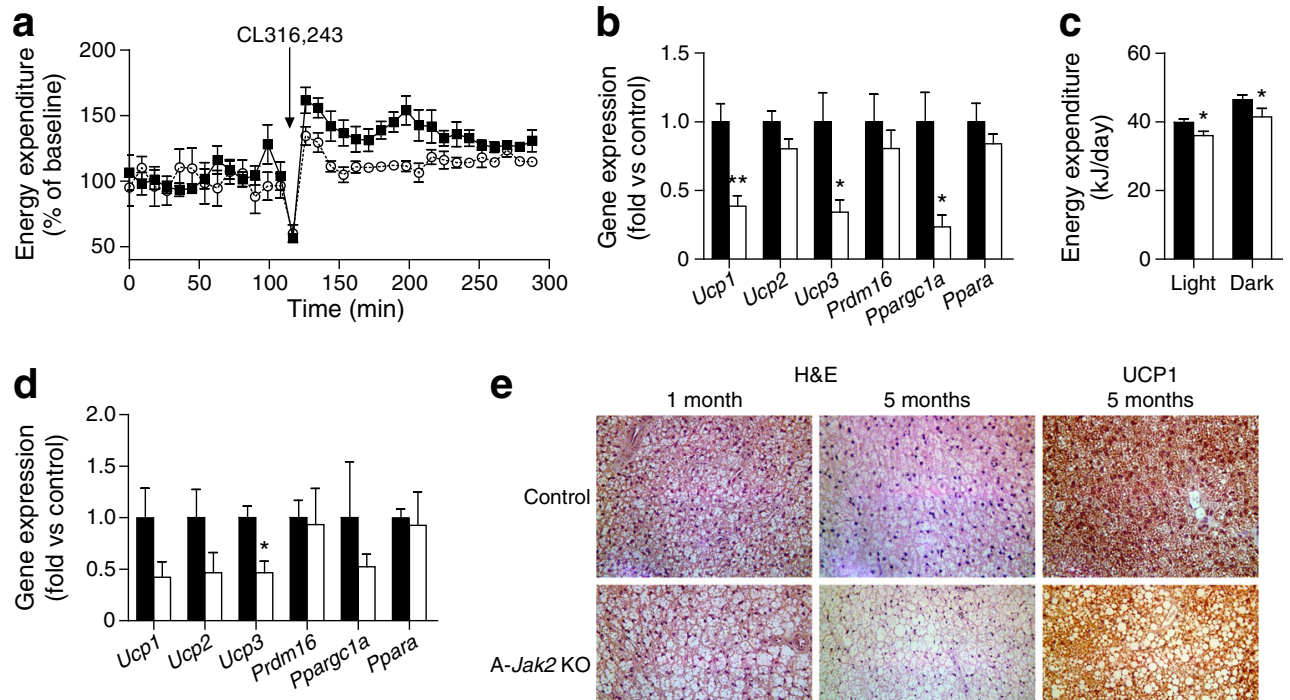

Fig. 2 Impaired BAT function in A-Jak2 KO mice. (a) Energy expenditure in chow-fed mice $(n=3-4)$. Results are presented as the percentage of baseline values, calculated as the average energy expenditure over the $2 \mathrm{~h}$ period before injection. Black, control; white, A-Jak2 KO. Overall $p<0.05$. (b) mRNA expression of genes in BAT and (c) energy expenditure in mice at 1 month of age $(n \geq 5)$. (d) mRNA expression of genes in BAT from chow-fed 5-month-old mice $(n=3)$. (e) Representative micrographs of H\&E and UCP1 immunostaining of BAT sections. Scale bar, $20 \mu \mathrm{m}$. Results are means \pm SEM. ${ }^{*} p<0.05$ and $* * p<0.01$ 
JAK2 is required for diet-induced thermogenesis in BAT Accordingly, with no difference in daily energy intake or respiratory exchange ratio (RER) on either chow or the HFD (Fig. 3a, b), control mice increased their energy expenditure in response to HFD feeding, whereas A-Jak2 $\mathrm{KO}$ mice did not (Fig. 3c-e), revealing an essential role for adipose JAK2 in diet-induced thermogenesis. Consistent with this, the HFD upregulated UCP1 in BAT in control, but not in A-Jak2 KO, mice (Fig. 3f, g). Conversely, in white adipose depots, HFD induced Ucpl to an equal extent in A-Jak2 $\mathrm{KO}$ mice and control littermates (Fig. 3f), suggesting that JAK2 is required for diet-induced UCP1 in BAT, but not in SAT or VAT. Expression patterns of other brown adipocyte markers in BAT were similar to that of $U c p 1$, showing a reduction in mRNA levels with Jak2 deficiency. Consistent with beige adipocytes being prominent in SAT, there was also a reduction in expression of brown/beige adipocyte markers in SAT of A-Jak2 KO mice compared with littermate controls, whereas no difference was observed in VAT between A-Jak2 KO and control mice (ESM Fig. 2a-c).

Recently, adipose resident immune cells were shown to be involved in thermogenesis $[16,33]$. To examine the possibility that JAK2 in immune cells plays a role in the observed phenotype of A-Jak2 KO mice, we measured JAK2 levels in the adipose stromal vascular fraction. We found no change in JAK2 protein abundance in the stromal vascular fraction of white adipose tissue or BAT from chow-fed A-Jak2 KO mice compared with control littermates (ESM Fig. 3a). In addition, while older A-Jak2 KO mice showed a higher macrophage content with M1 polarisation in their BAT (ESM Fig. 3b-d), the expression of macrophage markers was not significantly altered in BAT at 1 month of age when A-Jak2 KO mice and their control littermates had a similar body weight (ESM Fig. 3e). These results suggest that immune cell JAK2 probably does not contribute to the impaired BAT function observed in A-Jak2 KO mice.

To further investigate the mechanism of impaired BAT function, we examined JAK-STAT signalling in Jak2-deficient adipose tissue. Consistent with reduced JAK2mediated pathway activation, STAT5 phosphorylation was significantly attenuated in both BAT and white adipose tissue (Fig. 4). Levels of phospho-STAT3 were also reduced in BAT, suggesting that JAK2 is required for STAT3 activation in brown adipocytes. In contrast, STAT3 phosphorylation was significantly increased in SAT and VAT from A-Jak2 KO mice, probably as a compensatory mechanism. Together, these data suggest that STAT signalling in brown adipocytes requires JAK2 and may contribute to the regulation of UCP1 levels and BAT function.

Increased weight gain in HFD-fed A-Jak2 KO mice Accordingly, insufficient UCP1 induction upon HFD feeding resulted in lower energy expenditure in A-Jak2 $\mathrm{KO}$ mice (Fig. 5a-d). With no difference in RER or ambulatory activity (Fig. 5e, f), A-Jak2 KO mice gained significantly more weight on a HFD compared with controls (Fig. $5 \mathrm{~g}, \mathrm{~h}$ ). Analysis of fat depots indicated significant adipose tissue expansion in the SAT and VAT depots (Fig. 5i, j), with increased adipocyte size (Fig. 5k-m). Consistent with their increased adiposity, A-Jak2 $\mathrm{KO}$ mice displayed higher circulating leptin and resistin levels (Fig. 5n-q).

A-Jak2 KO mice develop HFD-induced insulin resistance and glucose intolerance We next determined whether impaired BAT function predisposed A-Jak2 $\mathrm{KO}$ mice to metabolic defects. HFD-fed male A-Jak2 KO mice had higher random and fasting blood glucose levels than control littermates (Fig. 6a, b). Furthermore, i.p. GTT indicated the
Fig. 3 JAK2 is required for dietinduced thermogenesis in BAT. (a) Daily energy intake, (b) RER, calculated as the ratio of carbon dioxide production volume to oxygen consumption volume $\left(\dot{V} \mathrm{O}_{2}\right),\left(\right.$ c) $\dot{V} \mathrm{O}_{2}$, (d) energy expenditure and (e) energy expenditure in HFD-fed mice analysed by ANCOVA $(n \geq 5$; $p<0.05)$. Black, control; white, A-Jak2 KO. (f) mRNA expression of $U c p 1(n \geq 5)$ in adipose tissues. (g) UCP1 protein levels in BAT $(n=3)$. Results are means \pm SEM. $* p<0.05$, ${ }^{* *} p<0.01,{ }^{* * *} p<0.001$ and ${ }^{\dagger} p=0.05$
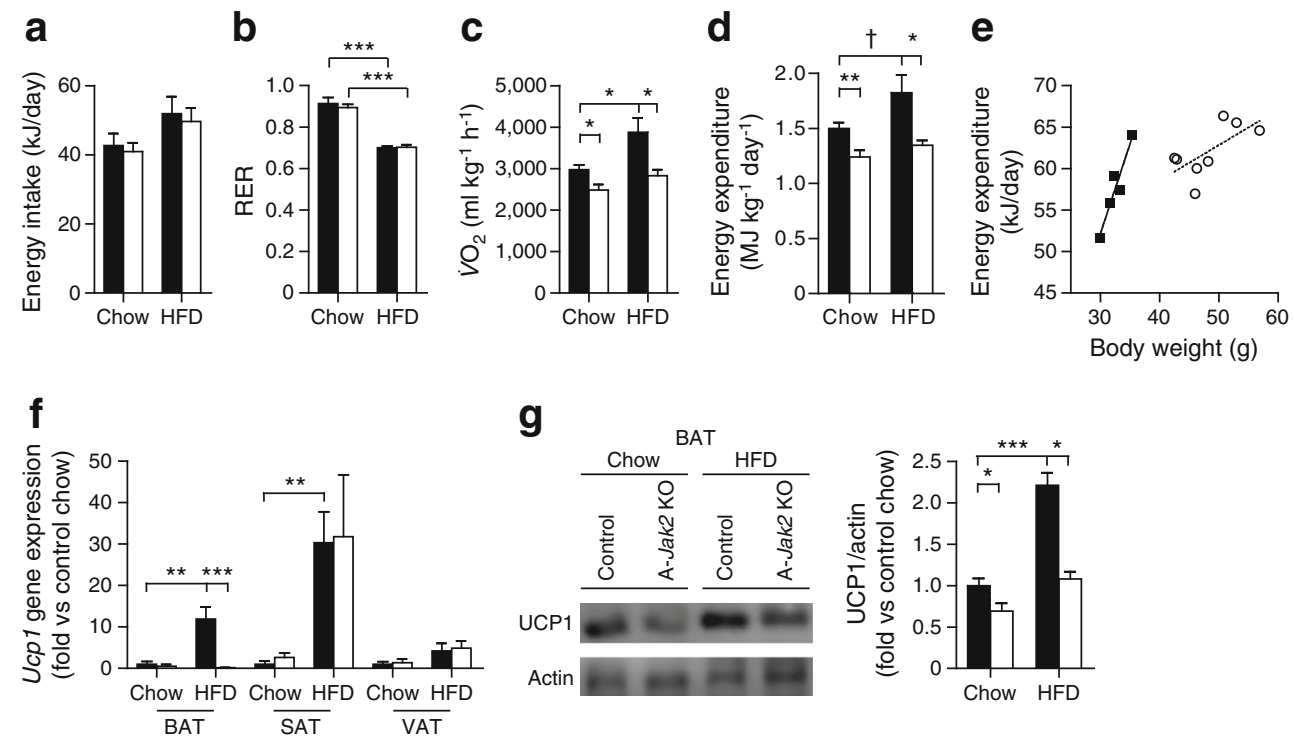


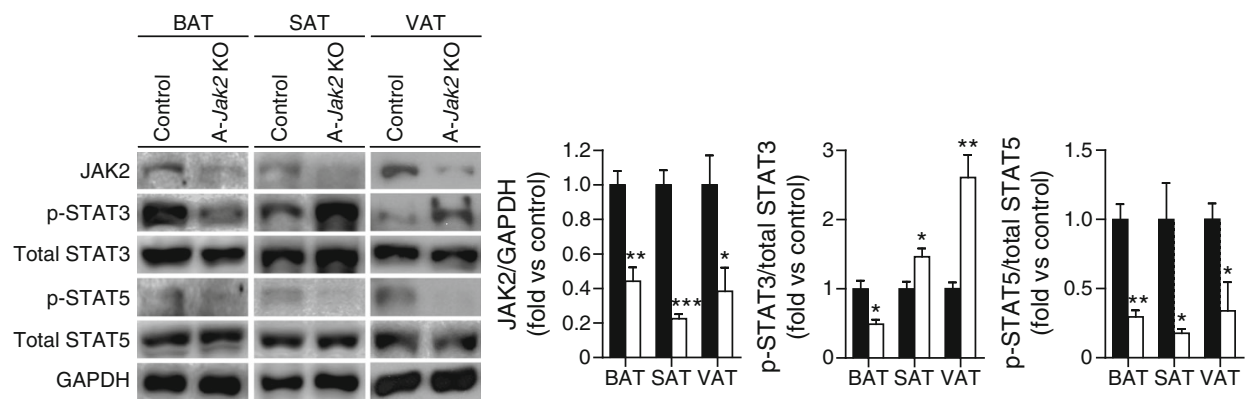

Fig. 4 Reduced STAT3 and STAT5 phosphorylation in BAT from AJak2 $\mathrm{KO}$ mice. Tissue lysates from HFD-fed mice were prepared and immunoblotted for JAK2, p-STAT3 (Y705) and p-STAT5 (Y694) $(n=$

3-5). Black, control; white, A-Jak2 KO. Results are means \pm SEM. ${ }^{*} p<0.05,{ }^{* *} p<0.01$ and ${ }^{* * *} p<0.001$

presence of impaired glucose tolerance (Fig. 6c). Male A-Jak2 KO mice also exhibited HFD-induced insulin resistance during an insulin tolerance test (ITT) compared with control litfemale A-Jak2 KO mice, although the degree of metabolic impairment was lower (Fig. 6e-h). Of note, disrupted glucose homeostasis in A-Jak2 $\mathrm{KO}$ mice may be secondary to their termates (Fig. 6d). Similar trends were observed in HFD-fed

higher body weight. Therefore, to specifically assess insulin

Fig. 5 Development of dietinduced obesity in A-Jak2 KO

mice. (a) $\dot{V} \mathrm{O}_{2}$; (b) energy expenditure; (c, d) ANCOVA analysis of energy expenditure during the (c) light and (d) dark cycle $(p<0.05)$; (e) RER; and (f) physical activity $(n \geq 6)$. Black, control; white, A-Jak2 KO. (g, h) Body weight of $(\mathbf{g})$ male $(n \geq 9)$ and (h) female $(n \geq 5)$ mice. (i) Absolute weight and (j) percentage total body weight of inguinal (Ing.), perigonadal (Peri.), retroperitoneal (Retro.), mesenteric (Mes.) and BAT fat pads $(n \geq 8)$. (k) Representative micrographs of H\&E staining of perigonadal adipose sections.

Scale bar, $50 \mu \mathrm{m}$. (1, m) Quantification of (l) adipocyte size distribution and (m) average adipocyte size $(n=3)$. $(\mathbf{n}-\mathbf{q})$

Serum adipokine levels $(n=7)$.

PAI-1, plasminogen activator inhibitor-1. Results are means \pm SEM. ${ }^{*} p<0.05,{ }^{*} p<0.01$ and $* * * p<0.001$
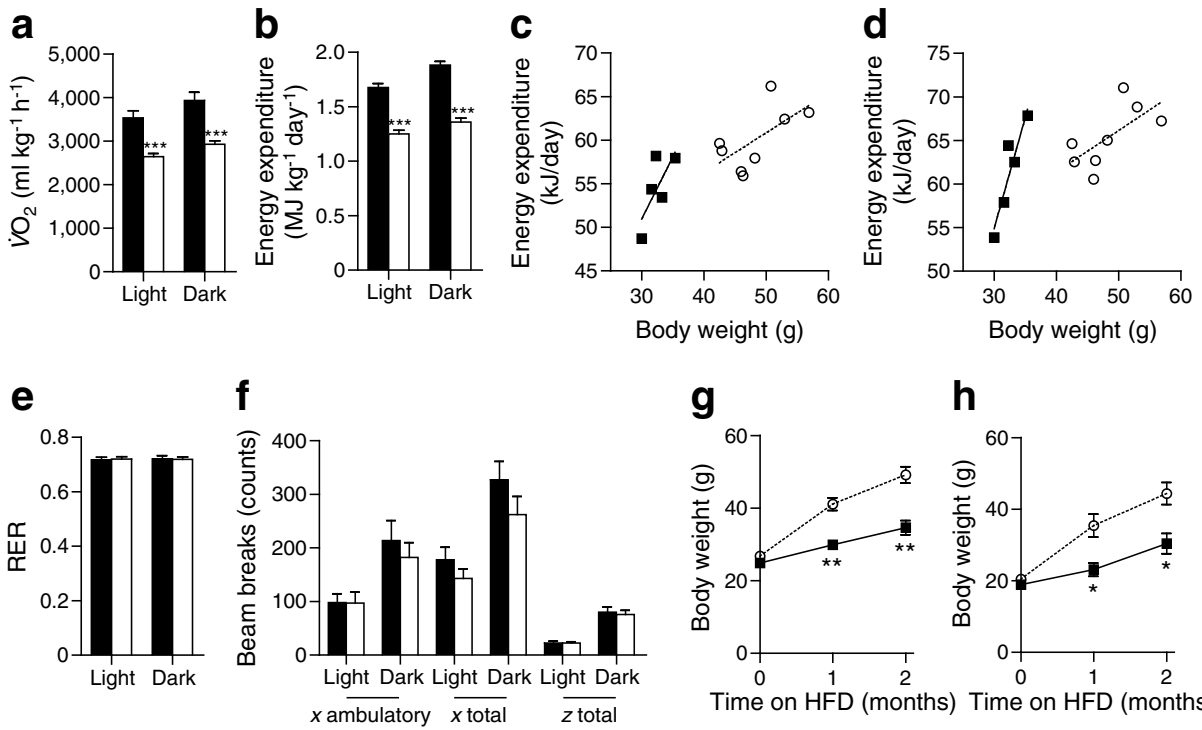

h
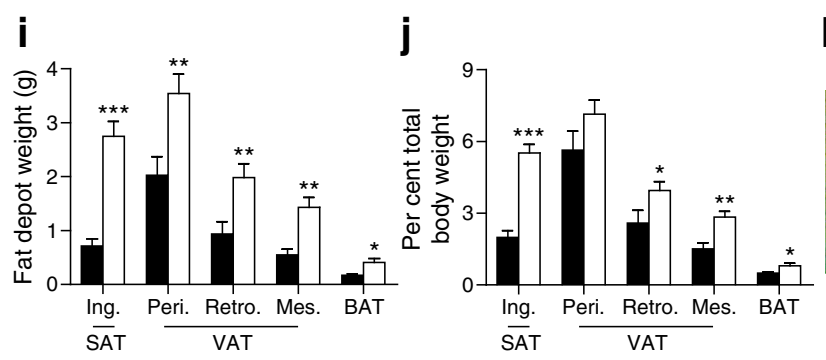

k
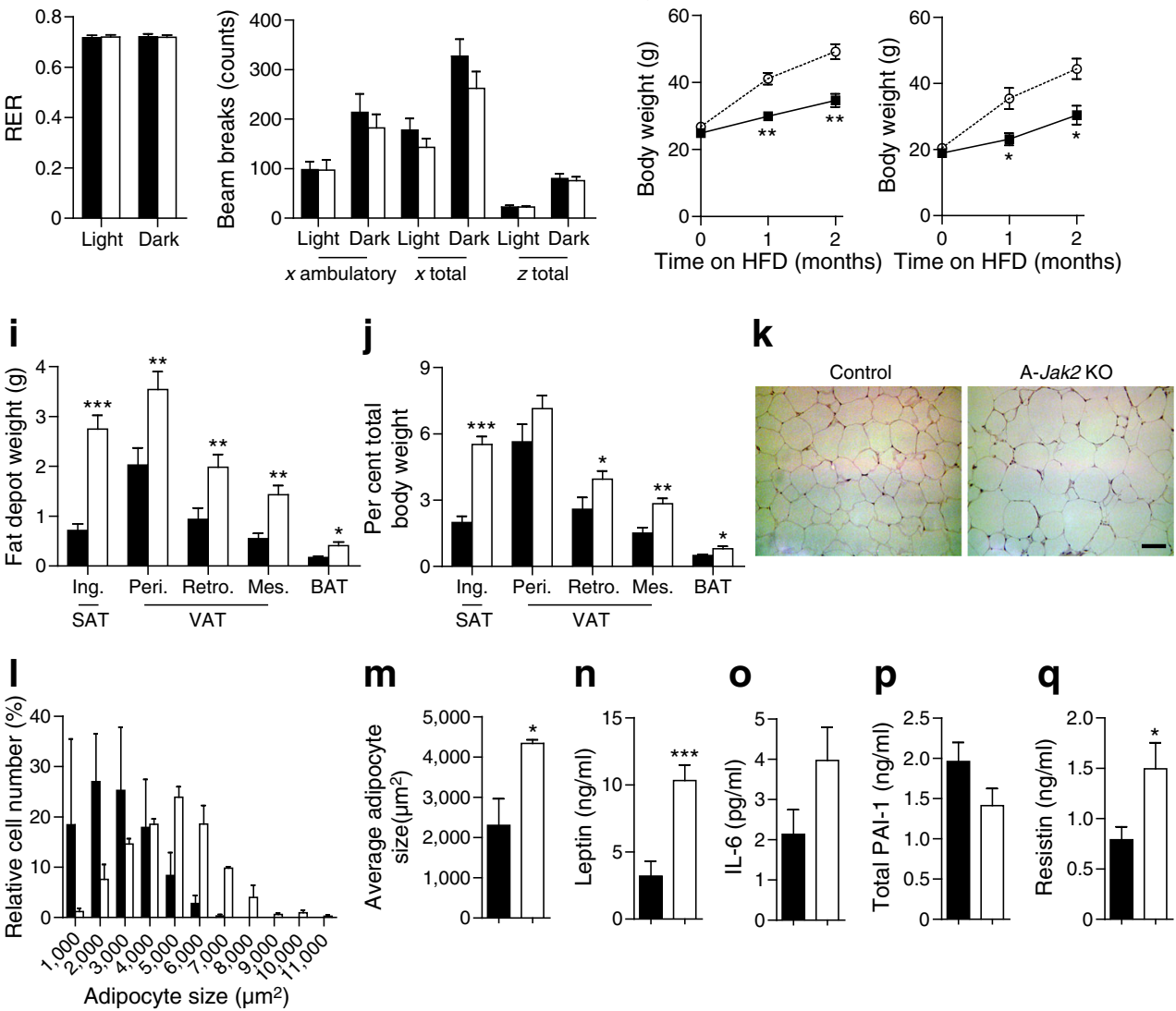

n $\quad 0$

O p
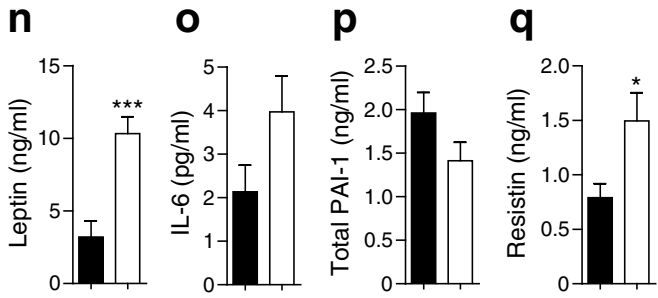
Fig. 6 HFD-induced insulin resistance and glucose intolerance in A-Jak2 $\mathrm{KO}$ mice. $(\mathbf{a}, \mathbf{e})$ Random blood glucose; $(\mathbf{b}, \mathbf{f})$ fasting blood glucose; $(\mathbf{c}, \mathbf{g})$ i.p. GTT $(1 \mathrm{~g} / \mathrm{kg})$; and $(\mathbf{d}, \mathbf{h})$ ITT $(1.0 \mathrm{U} / \mathrm{kg})$ in $(\mathbf{a}-\mathbf{d})$ male $(n \geq 8)$ and $(\mathbf{e}-\mathbf{h})$ female $(n \geq 5)$ mice. Inset: AUC (fold change over control). Black, control; white, A-Jak2 KO. (i) Tissue lysates were prepared from insulinstimulated $(5 \mathrm{U} / \mathrm{kg})$ female mice and immunoblotted for $\mathrm{p}$-Akt (S473) $(n=3)$. (j) Fasting serum insulin levels $(n \geq 5)$. Results are means \pm SEM. ${ }^{*} p<0.05$ and $* * p<0.01$
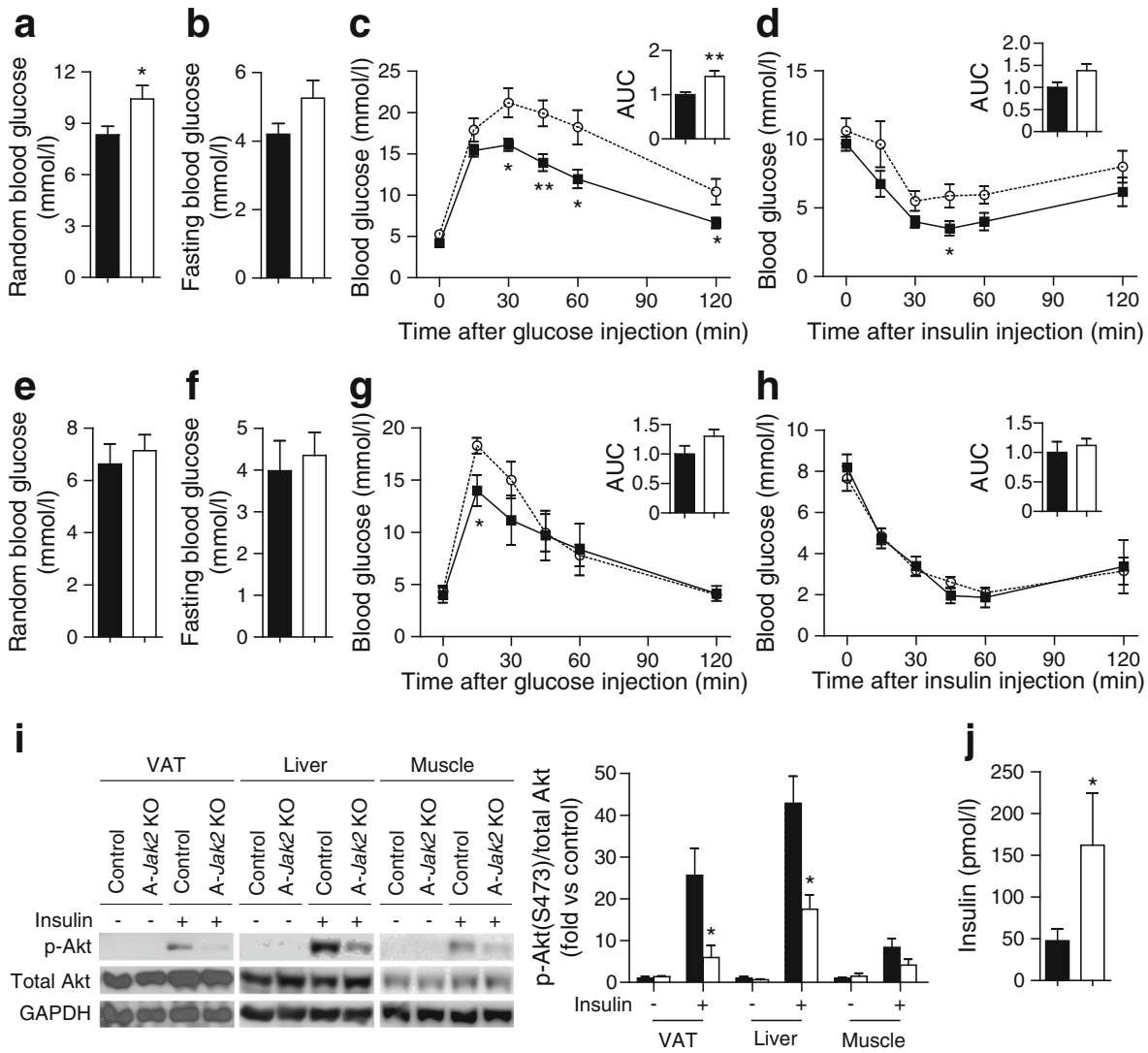

sensitivity in individual metabolic tissues, A-Jak2 $\mathrm{KO}$ mice and control littermates were injected with insulin and their tissues analysed for insulin signalling. As shown in Fig. 6i, HFD-fed A-Jak2 KO mice had reduced insulin-stimulated Akt phosphorylation in VAT, liver and skeletal muscle, indicating the presence of insulin resistance in these metabolic tissues that probably contributed to whole-body insulin resistance. Consistent with this notion, fasting serum insulin levels were significantly higher in A-Jak2 KO mice (Fig. 6j). Together, our results suggest that impaired BAT function in A-Jak2 KO mice predisposes them to HFD-induced obesity and associated metabolic disturbances.

\section{JAK2 is required for cold-induced thermogenesis in BAT} In addition to excess energy intake, cold exposure is another strong stimulator of non-shivering thermogenesis [34]. Therefore, we asked whether JAK2 is also regulated in response to cold. In contrast to metabolic stress, in which JAK2 was induced in all fat depots, acute thermal stress induced JAK2 in BAT only, and not in SAT or VAT (Fig. 7a, b). In parallel with JAK2, short-term cold challenge induced a modest elevation in UCP1 mRNA and protein levels in BAT (Fig. 7c-f). Intriguingly, BAT UCP1 levels paradoxically declined upon cold exposure in the absence of Jak2, indicating an essential role for JAK2 in the maintenance of UCP1 at a lower ambient temperature. Consistent with attenuated thermogenic activity, CS activity and the expression of brown adipocyte markers were significantly lower in BAT of A-Jak2 $\mathrm{KO}$ mice (Fig. $7 \mathrm{~g}$ and ESM Fig. 4a). Interestingly, cold challenge upregulated Ucp1 expression in white adipose depots from A-Jak2 KO mice, particularly in SAT (Fig. 7d, e), possibly as a compensatory mechanism to maintain the core body temperature. This was associated with higher CS activity and elevated expression of brown/beige adipocyte markers in SAT (Fig. 7h, i and ESM Fig. 4b, c). Nevertheless, this induction did not adequately activate thermogenesis. As a result, A-Jak2 $\mathrm{KO}$ mice were unable to maintain their body temperature after $3 \mathrm{~h}$ of cold exposure compared with control littermates (Fig. 7j). Together, these data indicate that JAK2 is essential for cold-induced UCP1 expression and thermogenesis in BAT.

\section{Discussion}

In this work, we studied the role of JAK2, a key player in cytokine signal transduction, in diet- and cold-induced thermogenesis. We showed that JAK2 expression in BAT is necessary for UCP1 induction in response to excess caloric intake. Consequently, mice with adipose-specific Jak2 disruption were not able to activate thermogenesis in response to an energy surplus and developed obesity, insulin resistance and glucose intolerance upon extended HFD feeding. JAK2 is also 
Fig. 7 Increased sensitivity to cold exposure in HFD-fed A-Jak2 KO mice. HFD-fed mice maintained at $20^{\circ} \mathrm{C}$ were placed at $4^{\circ} \mathrm{C}$ for $6 \mathrm{~h}(n=3)$. (a) mRNA expression and (b) protein levels of JAK2 in wild-type mice. Grey bars, $20^{\circ} \mathrm{C}$; hatched bars, $4^{\circ} \mathrm{C}$. (c-e) mRNA expression and (f) protein levels of UCP1. Black, control; white; A-Jak2 KO. (g-i) CS activity in tissue homogenates. (j) Body temperature measured at the indicated times. Results are means \pm SEM. ${ }^{*} p<0.05$, $* * p<0.01$ and $* * * p<0.001$ a

b

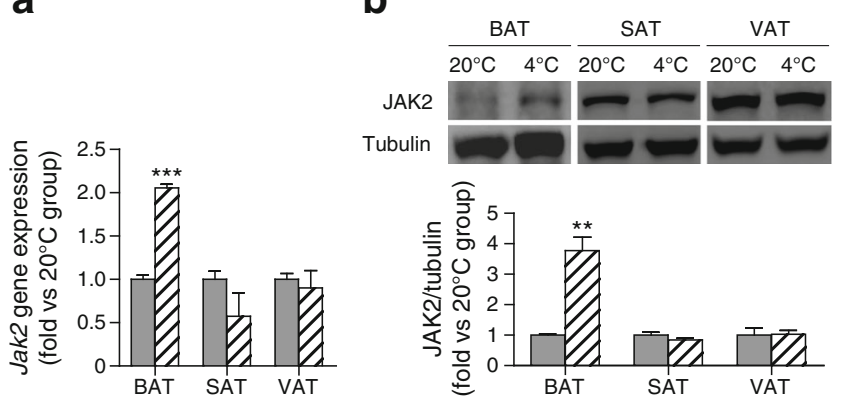

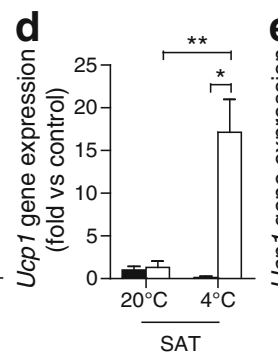

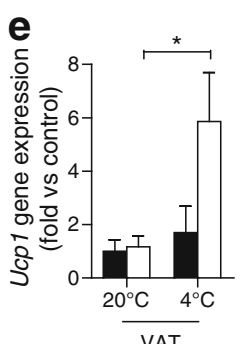

f
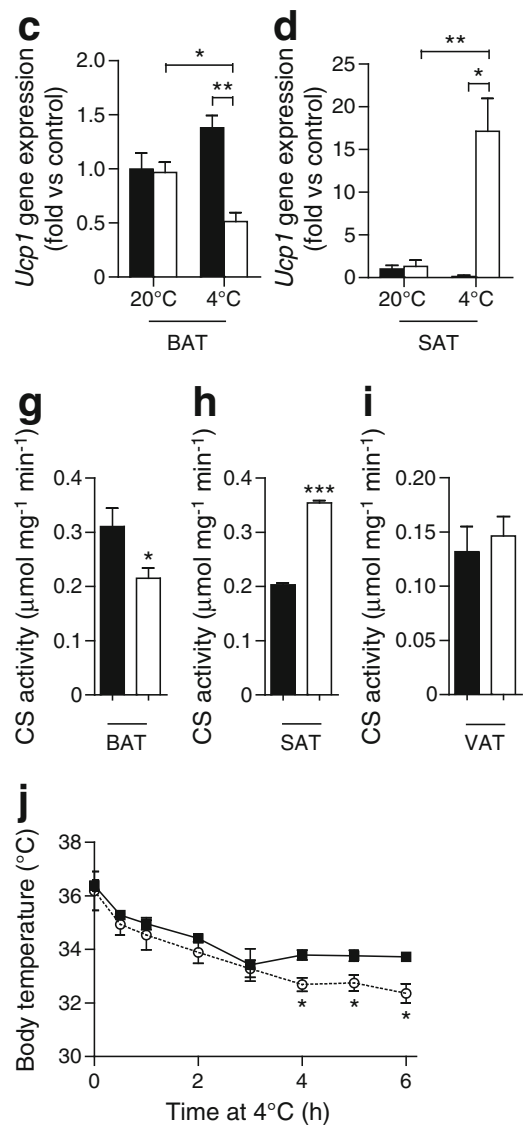

critical for non-shivering thermogenesis under thermal stress: A-Jak2 KO mice were cold intolerant.

Our observation that JAK2 expression is regulated by diet points to the possibility of pharmacological or dietary intervention to improve BAT function and thus treat obesity. While the HFD concomitantly induced JAK2 and UCP1 expression in all three fat depots examined, only BAT JAK2 appeared to be essential for UCP1 induction, whereas SAT and VAT upregulated UCP1 via a JAK2-independent mechanism. Thus, unlike in BAT, JAK2 is dispensable for the adaptive thermogenic response in white adipose tissue. These results highlight the unique properties and signalling requirements of the various fat depots for adaptive thermogenesis.

Our results suggest that JAK2 plays an important role in regulating $\mathrm{UCP} 1$ expression in $\mathrm{BAT}$ because A-Jak2 $\mathrm{KO}$ mice exhibited reduced UCP1 levels by as early as 1 month of age. Indeed, A-Jak2 KO mice were phenotypically similar to $U c p 1^{-/-}$mice, which are cold sensitive [31] and, when maintained at thermoneutrality, develop spontaneous obesity and show abolished diet-induced thermogenesis [35]. Of note, A-Jak2 KO mice were obese even when maintained at $20^{\circ} \mathrm{C}$. This difference compared with $U c p 1^{-/}$mice may be attributable to the suppression of lipolysis in white adipose tissue as a result of JAK2 deficiency, as we and others have previously shown $[25,36]$.

In this work, we showed that JAK2 is required for full activation of the thermogenic response to a $\beta_{3}$-adrenergic agonist, suggesting that JAK2 acts downstream of $\beta$-adrenergic signalling in adipocytes. This is consistent with the findings of our previous work [25]; together, they indicate that the actions 
of JAK2 mimic those of the sympathetic nervous system in adipose tissue, thus leading to the activation of lipolysis in white adipose tissue and stimulation of adaptive thermogenesis in BAT [37]. Indeed, in addition to the canonical signalling pathway, JAK-STAT signalling can be activated by G protein coupled receptors such as the angiotensin II type 1 receptor [38] and the chemokine receptors CCR2 [39] and CXCR4 [40]. Therefore, it is possible that the $\beta_{3}$-adrenergic receptor activates JAK2 in response to stimulation and transduces signals directly through the JAK-STAT cascade. The specific downstream targets of adrenergic receptor-activated JAKSTAT signalling remain to be elucidated.

In addition to crosstalk with adrenergic signalling, several hormones and growth factors could directly regulate BAT function via JAK2-STAT signalling. CNTF, leptin and GH all activate JAK2 and are known to regulate the expression of thermogenic genes [17, 20, 21, 24, 41]. The observed reduction in UCP1 expression in Jak2-deficient BAT could therefore be due to the concerted action of all these hormones.

The actions of JAK2-activating hormones are mostly mediated by STAT3 and STAT5 in metabolic tissues. In line with the disruption of upstream signal transduction, phosphorylation of both STAT3 and STAT5 is significantly attenuated in Jak2-deficient BAT. While the exact role of STAT5 in BAT remains to be elucidated, STAT3 has recently been implicated in BAT differentiation by binding to and enhancing protein stability of PR domain-containing protein 16 , a master regulator of brown and beige adipocyte differentiation [14]. Interestingly, in tissue-specific models of Jak2 disruption, STAT3 phosphorylation is reported to be upregulated [28], probably by other protein tyrosine kinases such as members of the Src family [42]. Indeed, we observed higher levels of phospho-STAT3 in white adipose depots from A-Jak2 KO mice compared with control littermates. On the other hand, STAT3 phosphorylation was significantly attenuated in Jak2deficient BAT, suggesting that JAK2 is necessary for STAT3 activation in this tissue.

Recently, JAK-STAT signalling in non-BAT has been implicated in the regulation of adaptive thermogenesis. For example, IL-4-stimulated activation of STAT6 in BAT macrophages promotes alternative $\mathrm{M} 2$ polarisation, which enhances macrophage secretion of catecholamines to sustain coldinduced thermogenesis [16]. We show here that $A p 2$-mediated recombination in our model did not affect JAK2 abundance in the adipose tissue stromal vascular fraction; thus, the contribution of JAK2 in resident immune cells to the observed phenotype of A-Jak2 $\mathrm{KO}$ mice is probably not significant.

In summary, our results uncovered a critical role for JAK2 in adipocyte biology and metabolic control. We showed that adipocyte JAK2 is required for UCP1 induction in BAT and diet- and cold-induced thermogenesis. Taken together, this work increases our understanding of the complex regulation of adaptive thermogenesis and suggests the potential of targeting JAK2 as a BAT activator to combat obesity and related metabolic disorders.

Acknowledgements We would like to thank K-U Wagner, University of Nebraska Medical Center, Omaha, NE, USA, for generously providing the Jak2-floxed mice and for assistance in genotyping.

Funding This work was supported by Canadian Institute of Health Research (CIHR) operating grant MOP-142193 and by a Canadian Diabetes Association (CDA) grant-in-aid to MW. MW is supported by the Canada Research Chair in Signal Transduction in Diabetes Pathogenesis. SYS and TS are supported by a CIHR Doctoral Research Award, a CDA Doctoral Student Research Award and a Canadian Liver Foundation Graduate Studentship. CTL is supported by the Eliot Phillipson Clinician Scientist Training Program, a CDA Postdoctoral Fellowship, a Canadian Society of Endocrinology and Metabolism Dr Fernand Labrie Fellowship Research Award and a Banting and Best Diabetes Centre (BBDC) Postdoctoral Fellowship. HRD is supported by a Queen Elizabeth II Graduate Scholarship in Science and Technology, a BBDC - University Health Network Graduate Award and a Department of Laboratory Medicine and Pathobiology Scholarship of Excellence.

Duality of interest statement The authors declare that there is no duality of interest associated with this manuscript.

Contribution statement SYS designed and performed the experiments, interpreted and analysed the data, and wrote and edited the manuscript; WZ, CTL, TS, JJB, SAS, HRD and AM performed the experiments, analysed data and edited the manuscript; MW designed the experiments, interpreted and analysed the data, and critically edited the manuscript. All authors read and approved the final version of the manuscript. MW is responsible for the integrity of the work as a whole.

\section{References}

1. Spiegelman BM, Flier JS (2001) Obesity and the regulation of energy balance. Cell 104:531-543

2. Cannon B, Nedergaard J (2004) Brown adipose tissue: function and physiological significance. Physiol Rev 84:277-359

3. Harms M, Seale P (2013) Brown and beige fat: development, function and therapeutic potential. Nat Med 19:1252-1263

4. Golozoubova V, Cannon B, Nedergaard J (2006) UCP1 is essential for adaptive adrenergic nonshivering thermogenesis. Am J Physiol Endocrinol Metab 291:E350-E357

5. Vitali A, Murano I, Zingaretti MC, Frontini A, Ricquier D, Cinti S (2012) The adipose organ of obesity-prone C57BL/6J mice is composed of mixed white and brown adipocytes. J Lipid Res 53: 619-629

6. Cypess AM, Lehman S, Williams G et al (2009) Identification and importance of brown adipose tissue in adult humans. N Engl J Med 360:1509-1517

7. Saito M, Okamatsu-Ogura Y, Matsushita M et al (2009) High incidence of metabolically active brown adipose tissue in healthy adult humans: effects of cold exposure and adiposity. Diabetes 58: $1526-1531$

8. Virtanen KA, Lidell ME, Orava J et al (2009) Functional brown adipose tissue in healthy adults. N Engl J Med 360:1518-1525

9. Rothwell NJ, Stock MJ (1979) A role for brown adipose tissue in diet-induced thermogenesis. Nature 281:31-35

10. Ouellet V, Labbe SM, Blondin DP et al (2012) Brown adipose tissue oxidative metabolism contributes to energy expenditure during acute cold exposure in humans. J Clin Invest 122:545-552 
11. Bachman ES, Dhillon H, Zhang CY et al (2002) betaAR signaling required for diet-induced thermogenesis and obesity resistance. Science 297:843-845

12. Young JB, Saville E, Rothwell NJ, Stock MJ, Landsberg L (1982) Effect of diet and cold exposure on norepinephrine turnover in brown adipose tissue of the rat. J Clin Invest 69:1061-1071

13. O'Shea JJ, Gadina M, Schreiber RD (2002) Cytokine signaling in 2002: new surprises in the Jak/Stat pathway. Cell 109(Suppl): S121-S131

14. Derecka M, Gornicka A, Koralov SB et al (2012) Tyk2 and Stat3 regulate brown adipose tissue differentiation and obesity. Cell Metab 16:814-824

15. Moisan A, Lee YK, Zhang JD et al (2015) White-to-brown metabolic conversion of human adipocytes by JAK inhibition. Nat Cell Biol 17:57-67

16. Nguyen KD, Qiu Y, Cui X et al (2011) Alternatively activated macrophages produce catecholamines to sustain adaptive thermogenesis. Nature 480:104-108

17. Hioki C, Yoshida T, Kogure A et al (2004) Effects of growth hormone $(\mathrm{GH})$ on mRNA levels of uncoupling proteins 1,2 , and 3 in brown and white adipose tissues and skeletal muscle in obese mice. Horm Metab Res 36:607-613

18. Li Y, Knapp JR, Kopchick JJ (2003) Enlargement of interscapular brown adipose tissue in growth hormone antagonist transgenic and in growth hormone receptor gene-disrupted dwarf mice. Exp Biol Med (Maywood) 228:207-215

19. Viengchareun S, Servel N, Feve B, Freemark M, Lombes M, Binart N (2008) Prolactin receptor signaling is essential for perinatal brown adipocyte function: a role for insulin-like growth factor-2. PLoS One 3:e1535

20. Siegrist-Kaiser CA, Pauli V, Juge-Aubry CE et al (1997) Direct effects of leptin on brown and white adipose tissue. J Clin Invest 100:2858-2864

21. Sarmiento U, Benson B, Kaufman S et al (1997) Morphologic and molecular changes induced by recombinant human leptin in the white and brown adipose tissues of C57BL/6 mice. Lab Invest 77: 243-256

22. Li G, Klein RL, Matheny M, King MA, Meyer EM, Scarpace PJ (2002) Induction of uncoupling protein 1 by central interleukin-6 gene delivery is dependent on sympathetic innervation of brown adipose tissue and underlies one mechanism of body weight reduction in rats. Neuroscience 115:879-889

23. Wernstedt I, Edgley A, Berndtsson A et al (2006) Reduced stressand cold-induced increase in energy expenditure in interleukin-6deficient mice. Am J Physiol Regul Integr Comp Physiol 291: R551-R557

24. Ott V, Fasshauer M, Dalski A, Klein HH, Klein J (2002) Direct effects of ciliary neurotrophic factor on brown adipocytes: evidence for a role in peripheral regulation of energy homeostasis. J Endocrinol 173:R1-R8

25. Shi SY, Luk CT, Brunt JJ et al (2014) Adipocyte-specific deficiency of Janus kinase (JAK) 2 in mice impairs lipolysis and increases body weight, and leads to insulin resistance with ageing. Diabetologia 57:1016-1026

26. Krempler A, Qi Y, Triplett AA, Zhu J, Rui H, Wagner KU (2004) Generation of a conditional knockout allele for the Janus kinase 2 (Jak2) gene in mice. Genesis 40:52-57
27. Wagner KU, Krempler A, Triplett AA et al (2004) Impaired alveologenesis and maintenance of secretory mammary epithelial cells in Jak2 conditional knockout mice. Mol Cell Biol 24: $5510-5520$

28. Shi SY, Garcia Martin R, Duncan RE et al (2012) Hepatocytespecific deletion of Janus kinase 2 (JAK2) protects against dietinduced steatohepatitis and glucose intolerance. J Biol Chem 287: 10277-10288

29. Wang L, Opland D, Tsai S et al (2014) Pten deletion in RIP-Cre neurons protects against type 2 diabetes by activating the antiinflammatory reflex. Nat Med 20:484-492

30. Choi D, Schroer SA, Lu SY et al (2010) Erythropoietin protects against diabetes through direct effects on pancreatic beta cells. J Exp Med 207:2831-2842

31. Enerback S, Jacobsson A, Simpson EM et al (1997) Mice lacking mitochondrial uncoupling protein are cold-sensitive but not obese. Nature 387:90-94

32. Lowell BB, S-Susulic V, Hamann A et al (1993) Development of obesity in transgenic mice after genetic ablation of brown adipose tissue. Nature 366:740-742

33. Lee MW, Odegaard JI, Mukundan L et al (2015) Activated type 2 innate lymphoid cells regulate beige fat biogenesis. Cell 160:74-87

34. Morrison SF, Madden CJ, Tupone D (2012) Central control of brown adipose tissue thermogenesis. Front Endocrinol (Lausanne) 3: pii: 00005

35. Feldmann HM, Golozoubova V, Cannon B, Nedergaard J (2009) UCP1 ablation induces obesity and abolishes diet-induced thermogenesis in mice exempt from thermal stress by living at thermoneutrality. Cell Metab 9:203-209

36. Nordstrom SM, Tran JL, Sos BC, Wagner KU, Weiss EJ (2013) Disruption of JAK2 in adipocytes impairs lipolysis and improves fatty liver in mice with elevated GH. Mol Endocrinol 27: $1333-1342$

37. Collins S, Surwit RS (2001) The beta-adrenergic receptors and the control of adipose tissue metabolism and thermogenesis. Recent Prog Horm Res 56:309-328

38. Marrero MB, Schieffer B, Paxton WG et al (1995) Direct stimulation of Jak/STAT pathway by the angiotensin II AT1 receptor. Nature 375:247-250

39. Mellado M, Rodriguez-Frade JM, Aragay A et al (1998) The chemokine monocyte chemotactic protein 1 triggers Janus kinase 2 activation and tyrosine phosphorylation of the CCR2B receptor. J Immunol 161:805-813

40. Vila-Coro AJ, Rodriguez-Frade JM, Martin De Ana A, MorenoOrtiz MC, Martinez AC, Mellado M (1999) The chemokine SDF1alpha triggers CXCR4 receptor dimerization and activates the JAK/STAT pathway. FASEB J 13:1699-1710

41. Commins SP, Watson PM, Padgett MA, Dudley A, Argyropoulos G, Gettys TW (1999) Induction of uncoupling protein expression in brown and white adipose tissue by leptin. Endocrinology 140:292-300

42. Turkson J, Bowman T, Garcia R, Caldenhoven E, De Groot RP, Jove R (1998) Stat3 activation by Src induces specific gene regulation and is required for cell transformation. Mol Cell Biol 18: $2545-2552$ 